\title{
EDITOR'S SPEAK
}

\section{Salivary Gland Disorders}

The management of salivary gland disorders has been shared by numerous clinical specialities namely otolaryngology, maxillofacial surgery, general surgery and oncosurgery, and while the treatment protocols may be fairly well defined, variations in presentation may occasioanlly mislead the clinician.

This is one of many, in a series of issues related to salivary glands with the attempt to bring together such knowledge on a common platform.

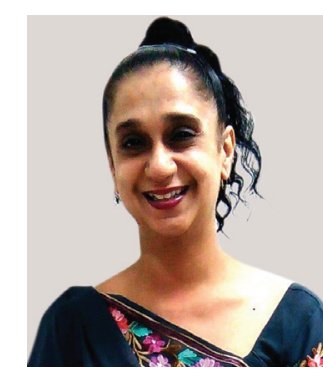

Bachi T Hathiram

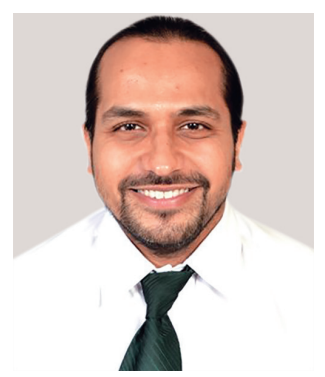

Vicky S Khattar

Editors-in-Chief

Bachi T Hathiram

Professor and Head

Department of ENT and Head and Neck Surgery

Topiwala National Medical College and

BYL Nair Charitable Hospital, Mumbai, Maharashtra, India

Visiting Consultant

Sir HN Reliance Foundation Hospital and

Medical Research Center, Mumbai, Maharashtra, India

Vicky S Khattar

Associate Professor

Department of ENT and Head and Neck Surgery

Topiwala National Medical College and

BYL Nair Charitable Hospital, Mumbai, Maharashtra, India

Visiting Consultant

Sir HN Reliance Foundation Hospital and Medical Research Center, Mumbai, Maharashtra, India 